\title{
Glycosphingolipid antigens from Leishmania (L.) amazonensis amastigotes. Binding of anti- glycosphingolipid monoclonal antibodies in vitro and in vivo
}

A.H. Straus ${ }^{1}$, V.B. Valero ${ }^{1}$, C.M. Takizawa ${ }^{1}$, S.B. Levery ${ }^{3}$, M.S. Toledo ${ }^{1}$, E. Suzuki ${ }^{1}$, M.E.K. Salyan ${ }^{4}$, S. Hakomori ${ }^{4}$, C.L. Barbieri ${ }^{2}$ and H.K. Takahashi ${ }^{1}$

\section{Correspondence}

A.H. Straus

Departamento de Bioquímica

Universidade Federal de

São Paulo/EPM

Rua Botucatu, 862

04023-900 São Paulo, SP

Brasil

Fax: 55 (011) 570-2509

E-mail: straus.bioq@epm.br

Research supported by FAPESP, $\mathrm{CNPq}$, and FINEP.

Received April 2, 1996

Accepted January 6, 1997

\author{
1Departamento de Bioquímica and 2Divisão de Parasitologia, \\ Escola Paulista de Medicina, Universidade Federal de São Paulo, 04023-900 \\ São Paulo, SP, Brasil \\ ${ }^{3}$ University of Georgia, Complex Carbohydrate Research Center, Athens, \\ GA 30602, USA \\ ${ }^{4}$ Pacific Northwest Research Foundation, Seattle, WA 98122 and \\ Department of Pathobiology and Microbiology, University of Washington, \\ Seattle, WA 98195, USA
}

\begin{abstract}
Specific glycosphingolipid antigens of Leishmania (L.) amazonensis amastigotes reactive with the monoclonal antibodies (MoAbs) ST-3, ST-4 and ST-5 were isolated, and their structure was partially elucidated by negative ion fast atom bombardment mass spectrometry. The glycan moieties of five antigens presented linear sequences of hexoses and $\mathrm{N}$-acetylhexosamines ranging from four to six sugar residues, and the ceramide moieties were found to be composed by a sphingosine d18:1 and fatty acids $24: 1$ or 16:0. Affinities of the three monoclonal antibodies to amastigote glycosphingolipid antigens were also analyzed by ELISA. MoAb ST-3 reacted equally well with all glycosphingolipid antigens tested, whereas ST-4 and ST-5 presented higher affinities to glycosphingolipids with longer carbohydrate chains, with five or more sugar units (slow migrating bands on HPTLC). Macrophages isolated from footpad lesions of BALB/c mice infected with Leishmania (L.) amazonensis were incubated with MoAb ST-3 and, by indirect immunofluorescence, labeling was only detected on the parasite, whereas no fluorescence was observed on the surface of the infected macrophages, indicating that these glycosphingolipid antigens are not acquired from the host cell but synthesized by the amastigote. Intravenous administration of ${ }^{125}$ I-labeled ST-3 antibody to infected BALB/c mice showed that MoAb ST-3 accumulated significantly in the footpad lesions in comparison to blood and other tissues.
\end{abstract}

Key words

- Glycosphingolipids

- Monoclonal antibodies

- Leishmania (L.) amazonensis

- Fast atom bombardment mass spectrometry

- Carbohydrate antigens 
We have recently described the isolation and structure of a new glycosphingolipid (GSL) antigen specific for Leishmania (L.) amazonensis amastigotes (1). This new antigen is a $\mathrm{III}^{3} \mathrm{~B}_{-} \mathrm{GalGb}_{3} \mathrm{Cer}$ (Gal-globoside) with the structure $\mathrm{Gal} \beta 1 \rightarrow 3 \mathrm{Gal} \alpha 1 \rightarrow$ $4 \mathrm{Gal} \beta 1 \rightarrow 4 \mathrm{Glc} \beta 1 \rightarrow \mathrm{Cer}$ (band 1), which differs from the human erythrocyte $\mathrm{P}$ antigen by the substitution of the terminal residue of GalNAcß1 $\rightarrow 3$ for Galß1 $\rightarrow 3(2,3)$. In addition to $\mathrm{III}^{3} \mathrm{~B}-\mathrm{GalGb}_{3} \mathrm{Cer}$, sixteen other GSL antigens (bands 2 to 17) were detected, most of them in very low amounts (4). We analyzed five antigens by negative ion fast atom bombardment mass spectrometry ( ${ }^{-} \mathrm{FAB} / \mathrm{MS}$ ) and determined their hexose and $\mathrm{N}$-acetyl hexose sequences as well as their ceramide moiety composition. ${ }^{-} \mathrm{FAB} / \mathrm{MS}$ was performed using a JEOL HX-110 mass spectrometer/DA-5000 data system (Jeol Ltd., Tokyo, Japan). Samples (5-10 $\mu$ g) were deposited on the target in a matrix of triethanolamine containing the cyclic polyether 15 crown-5 $(5,6)$ and bombarded with a $6-\mathrm{kV}$ Xenon beam source. The mass range $\mathrm{m} / \mathrm{z}$ 100-3600 was acquired in $1.5 \mathrm{~min}$ by linear upward scan, with an acceleration voltage of $10 \mathrm{kV}$ and a resolution of 3000 , using a 100$\mathrm{Hz}$ filter. Sodium iodide in glycerol was used as the calibration standard.

Of the seventeen antigenic GSLs isolated from $L$. (L.) amazonensis amastigotes (4), five glycosphingolipids, termed bands $\mathrm{b} 2$, b3, b4, b5 and b6, were purified by a combination of HPLC and preparative HPTLC in solvent chloroform/methanol/ $0.2 \%$ aqueous $\mathrm{CaCl}_{2}$, as described by Straus et al. (1) and analyzed by ${ }^{-} \mathrm{FAB} / \mathrm{MS}$. The MW of the pure GSL antigens b2, b3, b4, b5 and b6, recognized by the monoclonal antibodies (MoAbs) ST-3, ST-4, and ST-5 (directed against carbohydrate moieties of GSLs), was 1346 atomic mass units (a.m.u.), 1498 a.m.u., 1388 a.m.u., 1660 a.m.u. and 1550 a.m.u., respectively. The hexose and $\mathrm{N}$-acetyl hexose sequences of the bands analyzed were as follows: band 2: Hex-O-Hex-O-Hex-O-Hex-O-
Hex-O-Cer; band 3: Hex-O-HexNAc-O-Hex$\mathrm{O}-\mathrm{Hex}-\mathrm{O}-\mathrm{Hex}-\mathrm{O}-\mathrm{Cer}$; band 4: Hex-OHexNAc-O-Hex-O-Hex-O-Hex-O-Cer; band 5: Hex-O-Hex-O-HexNAc-O-Hex-O-Hex-OHex-O-Cer; and band 6: Hex-O-Hex-OHexNAc-O-Hex-O-Hex-O-Hex-O-Cer. Their ceramide moieties contained only one type of long chain base, a sphingosine d18:1, whereas their fatty acid (f.a.) composition was: band 2, f.a. 16:0; band 3, f.a. 24:1; band 4 , f.a. $16: 0$; band 5, f.a. $24: 1$ and band 6 , f.a. 16:0.

Eight different GSL antigens were purified as described above (bands 1, 3, 4, 5, 8, 9, 12, and 13) (Figure 1A), and analyzed by ELISA regarding their affinity for MoAbs ST-3, ST-4 and ST-5. The GSL antigens were double-diluted with the first well containing $100 \mathrm{ng}$ of the antigen $(1,7)$. MoAb ST-3 showed the same affinity for the eight GSL antigens tested. MoAb ST-4 showed higher affinity for bands 9,12 and 13 and lower affinity for bands $1,3,4,5$ and 8 . MoAb ST-5 presented higher affinity for glycosphingolipid antigens 4 to 13 and lower affinity for bands 1 and 3 . These data indicate that the fine specificity of these three MoAbs presents subtle differences depending on the structure and size of the glycan moiety of GSL antigens.

${ }^{125}$ I-labeled ST-3 antibody was used to determine the in vivo binding capacity of the anti-glycosphingolipid MoAbs. The radiolabeled MoAb was injected intravenously into $\mathrm{BALB} / \mathrm{c}$ mice infected with Leishmania (L.) amazonensis. After $72 \mathrm{~h}$ their blood was removed by cardiac puncture and the mice were sacrificed. Samples from the tissues and lesions were washed with cold hypotonic PBS (10-fold diluted PBS) and weighed and the radioactivity was counted. Tissue distribution of ${ }^{125}$ I-labeled ST-3 is reported as the ratio of radioactivity in the tissue to that present in the blood [(cpm/g tissue $) /$ (cpm/g blood)]. Antibody distribution was determined in three infected $\mathrm{BALB} / \mathrm{c}$ mice after injection of ${ }^{125} \mathrm{I}$-labeled antibody as 
described above. The data are shown in Figure $2 \mathrm{~A}$ and represent values obtained $72 \mathrm{~h}$ after injection of $3.0 \mu \mathrm{g}(10 \mu \mathrm{Ci})$ of ${ }^{125} \mathrm{I}-$ labeled ST-3. The highest level of radioactivity was observed in the footpad lesions, with almost three times more radioactivity being detected in the lesion when compared to blood and lung. An irrelevant antibody (CU-1) used as control (8) showed no sig- nificant binding.

The data presented in this study clearly show the importance of glycosphingolipid antigens recognized by MoAbs ST-3, ST-4 and ST-5 as specific markers of Leishmania (L.) amazonensis amastigotes. These glycosphingolipid antigens, different from those reported in amastigotes of Leishmania donovani and Leishmania mexicana (9), are
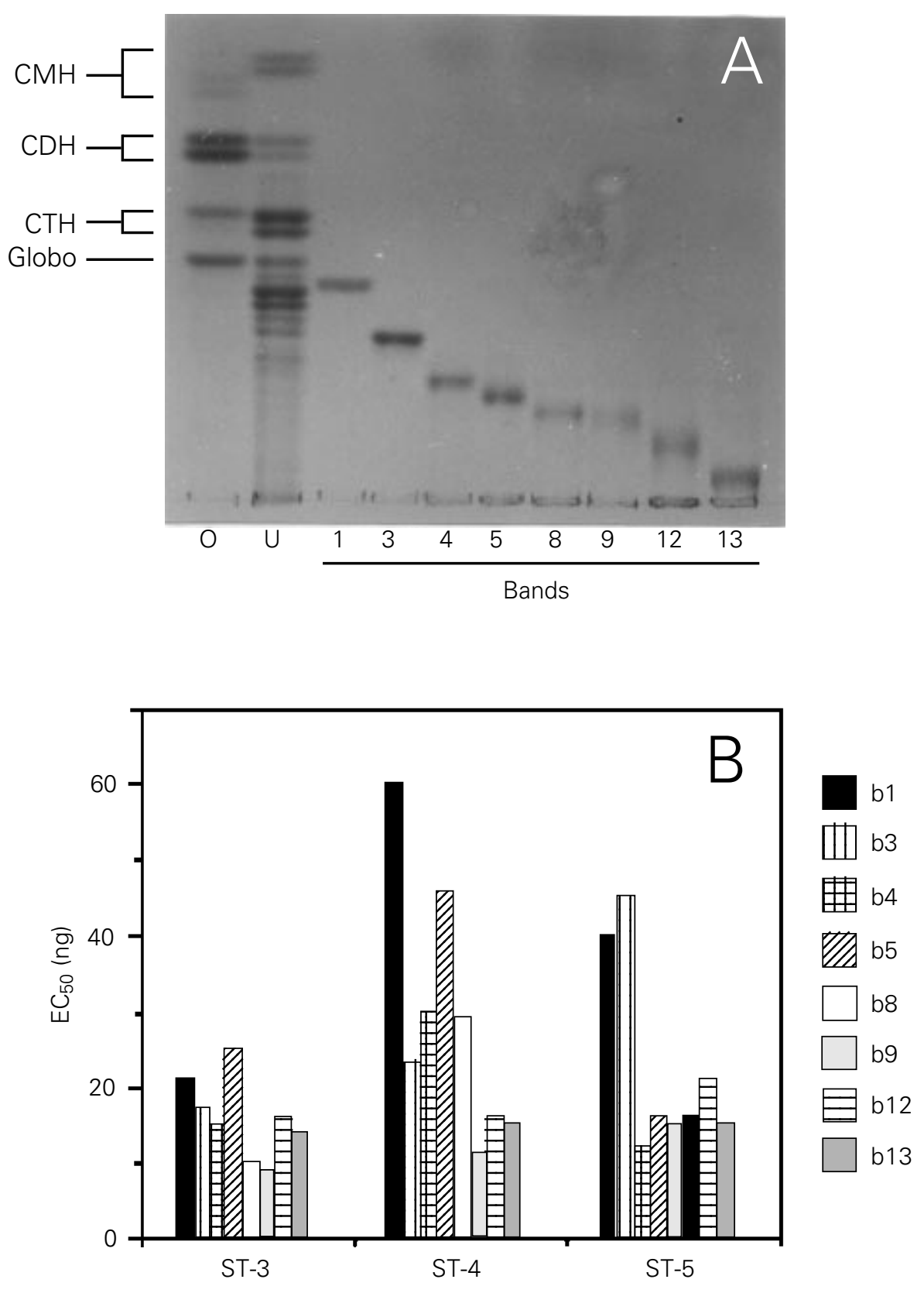

Figure 1 - Panel A, HPTLC pattern of 8 pure GSL antigens from L. (L.) amazonensis amastigotes developed in chloroform/methanol/aqueous $\mathrm{CaCl}_{2}$ (60:40:9; v/v/ v). $O$, Human erythrocyte neutral GSLs from Folch's lower phase; $U$, total neutral GSLs from $L$. (L.) amazonensis amastigotes. $\mathrm{CMH}$, Ceramide monohexoside; $\mathrm{CDH}$, ceramide dihexoside; $\mathrm{CTH}$, ceramide trihexoside; Globo, globoside. The plate was stained with orci$\mathrm{nol} / \mathrm{H}_{2} \mathrm{SO}_{4}$. Panel $B$, Affinity of MoAbs ST-3, -4 and -5 for eight GSL antigens (bands 1, 3, 4, 5, $8,9,12$ and 13). Antigens were serially double-diluted in ethanol from $100 \mathrm{ng} /$ well to $0.4 \mathrm{ng} /$ well and adsorbed on 96-well plates. Plates were blocked with $1 \%$ BSA/PBS (2 h) and incubated overnight at $4^{\circ} \mathrm{C}$ with the MoAbs. Antibody binding was determined after incubation with rabbit anti-mouse $\operatorname{lgG}(2 \mathrm{~h})$ and $10^{5} \mathrm{cpm}$ of 125 p protein $\mathrm{A}$ in $1 \%$ BSA (1 h). The radioactivity of each well was measured with a gamma counter and the results are reported as $\mathrm{EC}_{50}$ (glycosphingolipid antigen necessary to achieve $50 \%$ of maximum binding). 
Figure 2 - Panel A, Distribution of ${ }^{125}$ /-labeled monoclonal antibody ST-3 in tissues from infected BALB/c mice. Three infected BALB/c mice were injected with $3.0 \mu \mathrm{g}(10 \mu \mathrm{Ci})$ of 125/-labeled ST-3 MoAb. In control experiments, infected mice were injected with a labeled irrelevant MoAb (CU-1 MoAb) and sacrificed $72 \mathrm{~h}$ later. The radiolabeled MoAb distribution is reported as the mean \pm SEM ratio of radioactivity in the tissue to that present in the blood [/cpm/g tissue)/(cpm/g blood)]. Pane/ $B$, Immunofluorescence of the ST$3 \mathrm{MoAb}$ in a mouse lesion imprint. Mouse imprints were fixed with $2 \%$ formaldehyde, blocked with normal mouse serum (1:50) and incubated with fluoresceinisothiocyanate (FITC)-labeled ST3 MoAb. Only amastigotes inside the macrophages are reactive with MoAb ST-3. Bar $=20$ um. Panel C, Phase-contrast of a mouse lesion imprint.
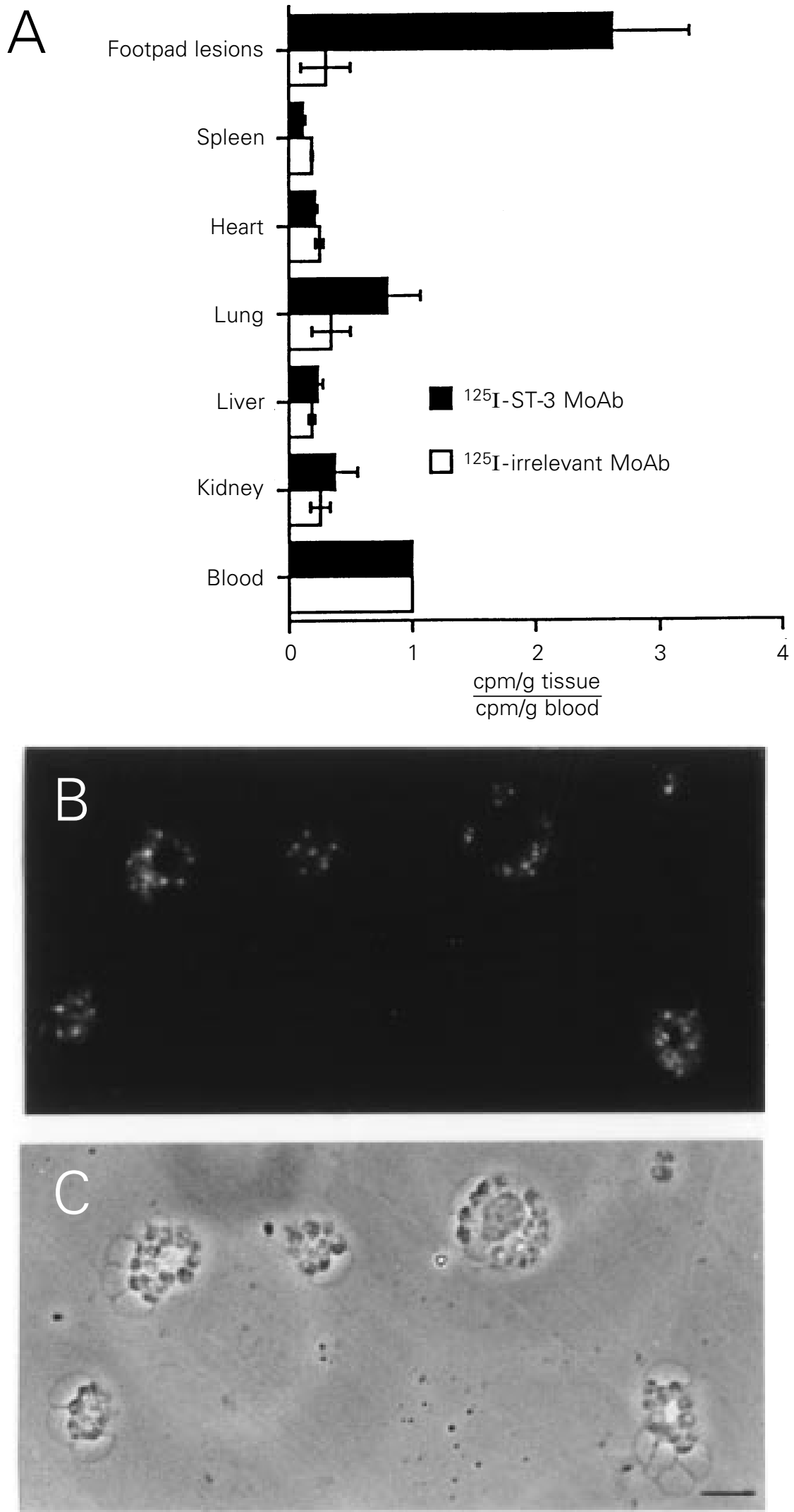
not acquired from the host (10) but rather synthesized by the parasite (1). Figure 2B shows MoAb ST-3 immunofluorescence of Leishmania(L.)amazonensis present in macrophages isolated from BALB/c mouse footpad lesions. Labeling was detected on the parasite and, as expected, no fluorescence was observed on the surface of the infected macrophages.

Anti-GSL monoclonal antibodies can greatly reduce the invasion of macrophages by the parasites. Nevertheless, no anti-GSL antibodies were detected in sera from patients with cutaneous leishmaniasis. Taken together, these data are consistent with the hypothesis that the lack of anti-GSL antibodies may render amastigote forms more effective in binding and invading host macrophages. Analysis by ${ }^{-} \mathrm{FAB} / \mathrm{MS}$ of five GSL antigens showed that they present linear carbohydrate structures and their ceramides range in mass from 536 a.m.u. to 646 a.m.u. Structural characterization of twelve other
GSL antigens is currently in progress. The complete structural elucidation of these antigens together with competitive assays using MoAbs ST-3, ST-4 or ST-5 may provide information concerning the fine specificity of these MoAbs as well as a more detailed vision of the GSL antigens directly related to binding/invasion of macrophages. One hypothesis currently under investigation in our laboratory is the presence of a unique UDP-galactose: Gal $\alpha 1 \rightarrow 4$ Gal $\beta 1 \rightarrow 3$ galactosyltransferase, responsible for the synthesis of Gal-globoside in these organisms using globotriosylceramide (Gal $\alpha 1 \rightarrow 4 \mathrm{Gal} \beta 1 \rightarrow$ $4 \mathrm{Glc} \beta 1 \rightarrow$ Cer) as substrate. The mechanism of precursor synthesis or transfer from the macrophage to the parasite is under investigation. Since Gal-globoside was found to be an amastigote-specific antigen which is not present in mammals, it may prove to be a new valuable target molecule for treatment and/or diagnosis of leishmaniasis caused by L. (L.) amazonensis.

\section{References}

1. Straus $A H$, Levery $S B$, Jasiulionis $M G$, Salyan ME, Steele SJ, Travassos LR, Hakomori S \& Takahashi HK (1993). Stagespecific glycosphingolipids from amastigote forms of Leishmania (L.) amazonensis. Journal of Biological Chemistry, 268: 13723-13730

2. Thorn JJ, Levery SB, Salyan MEK, Stroud MR, Cedergren B, Nilsson BE, Hakomori $S$ \& Clausen $H$ (1992). Structural characterization of $\mathrm{x}_{2}$ glycosphingolipid, its extended form, and its sialosyl derivatives: Accumulation associated with the rare blood group p phenotype. Biochemistry, 31: 6509-6517.

3. Rydberg L, Cedergren B, Breimer ME, Lindström K, Nyholm P-G \& Samuelson BE (1992). Serological and immunochemical characterization of anti-PP, $\mathrm{P}^{\mathrm{k}}$ (anti-TJa) antibodies in blood group little $p$ individuals. Blood group A type 4 recognition due to internal binding. Molecular Immunology, 29: 1273-1286.
4. Straus AH, Suzuki E, Toledo MS, Takizawa CM \& Takahashi HK (1995). Immunochemical characterization of carbohydrate antigens from fungi, protozoa and mammals by monoclonal antibodies directed to glycan epitopes. Brazilian Journal of Medical and Biological Research, 28: 919-923.

5. Fujii I, Isobe R \& Kanematsu K (1985). An easy and powerful technique of negative ion fast atom bombardment mass spectrometry employing a crown ether matrix. Journal of the Chemical Society, Chemical Communications, 7: 405-406.

6. Isobe R, Fujii I \& Kanematsu K (1987). Direct microanalysis by negative ion fast atom bombardment mass spectrometry. Trends in Analytical Chemistry, 6: 78-81.

7. Straus AH, Freymüller E, Travassos LR \& Takahashi HK (1996). Immunochemical and subcellular localization of the $43 \mathrm{kDa}$ glycoprotein antigen of Paracoccidioides brasiliensis with monoclonal antibodies. Journal of Medical and Veterinary Mycology, 34: 181-186.
8. Takahashi HK, Metoki R \& Hakomori S (1988). Immunoglobulin $G_{3}$ monoclonal antibody directed to $\mathrm{Tn}$ antigen (tumorassociated $\alpha$-N-acetylgalactosaminyl epitope) that does not cross-react with blood group A antigen. Cancer Research, 48: 4361-4367.

9. Winter G, Fuchs M, McConville MJ, Stierhof Y-D \& Overath P (1994). Surface antigens of Leishmania mexicana amastigotes: characterization of glycoinositol phospholipids and a macrophage-derived glycosphingolipid. Journal of Cell Science, 107: 2471-2482.

10. McConville MJ \& Blackwell JM (1991) Developmental changes in the glycylated phosphatidylinositols of Leishmania donovani. Journal of Biological Chemistry, 266: 15170-15179. 